\title{
Obtenção da Massa Molar de Asfaltenos através de Osmometria de Pressão de Vapor
}

\author{
Lyzette G. M. de Moura, Antonio Carlos S. Ramos \\ Departamento de Tecnologia Química, UFMA
}

\begin{abstract}
Resumo: A massa molar é uma propriedade essencial na caracterização de asfaltenos e um dos principais parâmetros de entrada nos modelos para a predição da precipitação. Na literatura são relatadas massas molares entre 1000 e 10000 g.mol ${ }^{-1}$ para os asfaltenos, variando em função da técnica, natureza do petróleo, tipo de solvente e temperatura. Neste trabalho foi determinada a massa molar média numérica para dois asfaltenos em tolueno, o C7I (insolúveis em heptano) e o C5I (insolúveis em pentano) através da osmometria de pressão de vapor. Os dados experimentais foram avaliados levando em consideração efeitos da agregação dos asfaltenos em solução e sua maior dispersão em baixas concentrações. Foram feitos ainda ajustes matemáticos respeitando a tendência das curvas para diluições infinitas buscando produzir melhores resultados no valor da massa molar. Os valores obtidos foram comparados com os métodos convencionais aplicados à análise da osmometria de pressão de vapor, e situaram-se entre 3200 e 5200 g.mol ${ }^{-1}$ para o asfaltenos C5I e entre 4100 e 5400 g.mol ${ }^{-1}$ para o C7I.
\end{abstract}

Palavras-chave: Massa molar, osmometria de pressão de vapor, asfaltenos, auto-associação.

\section{Determination of the Molar Mass of Asphaltenes Using Vapor Pressure Osmometry}

Abstract: Molar mass is an essential property for the characterization of asphaltenes and one of the main input parameters in the models for the prediction of the precipitation. In the literature molar masses between 1,000 and 10,000 g.mol ${ }^{-1}$ for the asphaltenes are quoted, depending on the technique, petroleum origin, solvent nature and temperature. In this work the numerical average molar mass for two asphaltenes in toluene, the C7I (insoluble in heptane) and the C5I (insoluble in pentane), was determined by vapor pressure osmometry. The experimental data were evaluated taking into account effects of asphaltenes aggregation in solution and its larger dispersion at low concentrations. Mathematical fittings were also made to comply with the curve bias for infinite dilutions, which was aimed at finding more accurate values for the molar mass. The results found were compared with the conventional methods applied to the analysis of the vapor pressure osmometry and varied from 3,200 to 5,200 g.mol-1 for the C5I asphaltenes and from 4,100 to 5,400 g.mol ${ }^{-1}$ for C7I.

Keywords: Molar mass, vapor pressure osmometry, asphaltenes, self-association.

\section{Introdução}

O petróleo consiste em uma mistura complexa de hidrocarbonetos que podem ser divididos de acordo com diversos critérios, como, por exemplo, a polaridade, que os classifica em asfaltenos, resinas, aromáticos e saturados ${ }^{[1]}$. Dentre estes, asfaltenos e resinas destacam-se por serem macromoléculas com tendência à auto-associação, o que as torna particularmente importantes para a indústria do petróleo, pois durante as diversas etapas de produção e processamento, podem tornar-se instáveis devido a variações de composição, temperatura e pressão, causando diversos problemas operacionais $^{[2,3]}$.

Os principais problemas relacionados aos asfaltenos são ocasionados pela floculação; formação e estabilização de emulsões e espumas; alterações na molhabilidade original do reservatório; e, principalmente, pela deposição, que consiste tipicamente em uma precipitação seguida de sedi- mentação. Tais problemas podem reduzir a produtividade, aumentando os custos do processo ${ }^{[4-6]}$.

O Instituto do Petróleo, Londres (UK), no Standard methods for analysis and testing of petroleum and related products (1989) define asfaltenos, através do método IP143/84 $4^{[7]}$, como sólidos amorfos, de coloração entre marrom escuro e preto, precipitados pela adição de excesso de n-heptano e solúveis em tolueno ou benzeno, a quente. Este procedimento, na prática, é empregado para a separação de frações distintas de asfaltenos, tais como C5I (insolúveis em pentano) e C7I (insolúveis em heptano) ${ }^{[4,8]}$. Como se trata de sistemas polidispersos é esperado que cada fração possua massa molar média com valores diferentes.

A massa molar constitui uma propriedade útil na caracterização dos asfaltenos e representa um parâmetro de entrada nos modelos que descrevem sua precipitação em diferentes petróleos. Diversas técnicas são utilizadas para determinar massas molares dos asfaltenos, tais como osmometria de

Autor para correspondência: Antonio Carlos S. Ramos, Departamento de Tecnologia Química, Centro de Ciências Exatas e Tecnologia, Universidade 
pressão de vapor, espectrometria de massa, espalhamento de luz, técnicas cromatográficas e viscosimetria. Em geral, os resultados apresentam limitações que podem ser relacionadas, sobretudo, com a natureza complexa destes sistemas ${ }^{[9]}$.

Os métodos baseados em propriedades coligativas, como a osmometria de pressão de vapor, fornecem uma massa molar média numérica, $\overline{\mathrm{M}}_{\mathrm{n}}$ (Equação 1):

$$
\overline{\mathrm{M}}_{\mathrm{n}}=\frac{1}{\mathrm{~N}} \sum_{\mathrm{i}} \mathrm{n}_{\mathrm{i}} \mathrm{M}_{\mathrm{i}}
$$

Em que $\mathrm{N}$ é a quantidade de matéria de uma amostra (em mol), $n_{\mathrm{i}}$ é a quantidade de matéria de cada espécie molecular e $\mathrm{M}_{\mathrm{i}}$ é a massa molar de cada espécie.

$\mathrm{Na}$ literatura podem ser encontrados valores de massa molar de asfaltenos bastante distintos, variando entre 1000 e 10000 g.mol ${ }^{-1}$,o que pode ser justificado, basicamente, pela origem do petróleo; natureza do solvente empregado nas determinações experimentais ${ }^{[10]}$; temperatura ${ }^{[9-12]}$ e método de determinaçãa ${ }^{[12,13]}$.

Diversos trabalhos têm aplicado a osmometria de pressão de vapor (OPV) para determinações de massa molar de asfaltos e asfaltenos ${ }^{[9,14-17]}$, pois se trata de uma técnica simples e de baixo custo $^{[18]}$, apesar de os valores variarem em relação à natureza do solvente empregado ${ }^{[12]} \mathrm{e}$ à temperatura de determinação ${ }^{[19-21]}$.

Trabalhos recentes ${ }^{[22-24]}$ apresentaram uma forte crítica ao emprego de OPV para a determinação da massa molar de asfaltenos, sobretudo porque estas frações apresentam tendência à formação de agregados mesmo em baixas concentrações. Como a técnica baseia-se em propriedades coligativas, o sinal obtido é proporcional ao número de partículas em solução independentemente do estado de agregação (dímeros, trímeros e micelas, por exemplo) ${ }^{[24]}$.

Diversos trabalhos evidenciaram a tendência auto-associativa dos asfaltenos em sistemas modelo empregando diferentes técnicas, tais como SAXS, SANS ${ }^{[25,26]}$, medidas de tensão superficial/interfacial ${ }^{[27,28]}$, calorimetria ${ }^{[29]}$ e cromatografia por exclusão de tamanho ${ }^{[30]}$. Alguns trabalhos indicaram a formação de nanoagregados em concentrações menores $\left(50 \text { a } 150 \mathrm{mg} . \mathrm{L}^{-1}\right)^{[31-33]}$ que aquelas utilizadas nos ensaios de osmometria de pressão de vapor. Devido isso, Mullins et al. ${ }^{[24]}$, ressaltam que os valores obtidos a partir de medidas de OPV podem exceder de 5 a 10 vezes os obtidos através de outras técnicas. Recentemente outros procedimentos foram aplicados na determinação da massa molar dos asfaltenos, tais como pela medida de coeficiente de difusão dos asfaltenos pelo método de dispersão de Taylor ${ }^{[34]}$, através de espectroscopia de correlação de fluorescência ${ }^{[35,36]}$, ressonância magnética nuclear ${ }^{[37]}$ e despolarização da fluorescência resolvida no tempo ${ }^{[22]}$. Em geral, nos trabalhos atuais, verifica-se uma tendência em produzir valores menores para a massa molar dos asfaltenos de diversas origens comparados aos obtidos pela técnica de OPV ${ }^{[38]}$. Porém, dados de espectrometria de massa mostraram uma boa concordância com os valores de massa molar dos asfaltenos obtidos pela técnica de OPV ${ }^{[18]}$.

A partir de medidas de tensão superficial/interfacial, Mohamed et al. (1999) ${ }^{[4]}$ determinaram a concentração micelar crítica (CMC) para dois asfaltenos em solventes como tolueno, piridina e nitrobenzeno. Aplicando os dados de tensão superficial na isoterma de Gibbs, foi possível uma estimativa do tamanho molecular, que se revelou consistente com os valores de massa molar desses mesmos sistemas obtidos através de osmometria de pressão de vapor. Resultados equivalentes foram obtidos também por Rogel et al. ${ }^{[28]}$.

Vazquez \& Mansoori ${ }^{[39]}$ determinaram a massa molar para três tipos de asfaltenos empregando a técnica de GPC e observaram uma distribuição contínua com um valor médio de 7747 g.mol ${ }^{-1}$ para os asfaltenos C9I, enquanto que para os asfaltenos C5I e C7I foram observadas duas populações distintas $\left(6751\right.$ e 2663 g.mol ${ }^{-1}$ para o C5I e 9383 e 3383 g. mol $^{-1}$ para o C7I). Tal resultado caracteriza a alta polidispersidade desses sistemas, revelando ainda que uma quantidade significativa de moléculas de resinas, sem caráter agregativo, constitui a fração asfaltênica. Nos trabalhos de $\operatorname{Ramos}^{[5]}$ a maior quantidade de resinas na fração C5I foi interpretada como responsável pela menor tendência a agregação destes asfaltenos frente aos asfaltenos C7I. Contudo, a formação de micelas foi observada apenas em concentrações mais elevadas 5,0 a 14,0 g.L $\mathrm{L}^{-1}$ em função do tipo de asfaltenos e do meio (tolueno, piridina e nitrobenzeno).

Neste trabalho efetuou-se a determinação da massa molar de asfaltenos C5I e C7I em tolueno, através da técnica de osmometria de pressão de vapor, em que os sistemas foram varridos em uma faixa ampla de forma a englobar a concentração micelar crítica. Cuidados foram tomados na parte experimental e no tratamento dos dados objetivando valores de massa molar dos asfaltenos mais confiáveis.

\section{Descrição do Equilíbrio Líquido-Vapor para Solução com Soluto não Volátil}

Para um sistema líquido-vapor no equilíbrio, em que o líquido consiste de uma solução contendo um soluto não volátil, além de temperatura e pressão, a fugacidade da fase vapor $\left(f^{\vee}\right)$ deve ser igual à da fase líquida $\left(\hat{f}^{\mathrm{L}}\right)^{[40]}$ :

$$
\mathrm{d} \ln f^{\mathrm{v}}=\mathrm{d} \ln \hat{f}^{\mathrm{L}}
$$

Expandindo-se esses termos em função de temperatura (T) e pressão $(\mathrm{P})$ para o vapor puro e de temperatura, pressão e composição (x) para a solução, obtém-se:

$$
\begin{aligned}
& \left(\frac{\partial \ln f^{\mathrm{V}}}{\partial \mathrm{T}}\right)_{\mathrm{P}} \mathrm{dT}+\left(\frac{\partial \ln f^{\mathrm{V}}}{\partial \mathrm{P}}\right)_{\mathrm{T}} \mathrm{dP}=\left(\frac{\partial \ln \hat{f}^{\mathrm{L}}}{\partial \mathrm{T}}\right)_{\mathrm{P}, \mathrm{x}} \\
& \mathrm{dT}+\left(\frac{\partial \ln \hat{f}^{\mathrm{L}}}{\partial \mathrm{P}}\right)_{\mathrm{T}, \mathrm{x}} \mathrm{dP}+\left(\frac{\partial \ln \hat{f}^{\mathrm{L}}}{\partial \mathrm{x}}\right)_{\mathrm{T}, \mathrm{P}} \mathrm{dx}
\end{aligned}
$$


Da relação entre potencial químico, fugacidade e energia molar de Gibbs obtém-se a variação da fugacidade com a pressão, tanto da fase vapor quanto da fase líquida, genericamente:

$$
\left(\frac{\partial \ln f}{\partial \mathrm{P}}\right)_{\mathrm{T}}=\frac{\mathrm{V}}{\mathrm{RT}}
$$

Para a determinação do efeito da temperatura sobre a fugacidade, duas equações devem ser consideradas. A primeira equação expressa a energia de Gibbs como:

$$
\mathrm{G}=\mathrm{H}-\mathrm{TS} \Rightarrow \frac{\partial}{\partial \mathrm{T}}\left(\frac{\mathrm{G}}{\mathrm{T}}\right)_{\mathrm{P}, \mathrm{x}}=\frac{\partial}{\partial \mathrm{T}}\left(\frac{\mathrm{H}}{\mathrm{T}}-\mathrm{S}\right)_{\mathrm{P}, \mathrm{x}}=-\frac{\mathrm{H}}{\mathrm{T}^{2}}
$$

A segunda equação representa a diferença entre as propriedades $\mathrm{T}, \mathrm{V}$ ou $\mathrm{T}, \mathrm{P}$ em um gás real e $\mathrm{T}, \mathrm{V}^{0}$ em um gás ideal, em que $\mathrm{V}^{0}=\mathrm{RT} / \mathrm{P}$ :

$$
\mathrm{G}-\mathrm{G}^{0}=\mathrm{RT} \ln \frac{f}{\mathrm{P}}
$$

Tomando-se $f / \mathrm{P}=f^{\mathrm{v}}$, no caso da fase vapor, dividindo-se a Equação 6 por RT e diferenciando em relação à temperatura sob pressão constante, obtém-se nova equação que, combinada com a Equação 5, resulta:

$$
\left[\frac{\partial \ln \left(f^{\mathrm{V}}\right)}{\partial \mathrm{T}}\right]_{\mathrm{P}}=-\frac{\mathrm{H}-\mathrm{H}^{0}}{\mathrm{RT}^{2}}
$$

Ou, levando-se em consideração a composição (x), no caso de uma mistura:

$$
\left[\frac{\partial \ln \left(\hat{f}^{\mathrm{L}}\right)}{\partial \mathrm{T}}\right]_{\mathrm{P}, \mathrm{x}}=-\frac{\overline{\mathrm{H}}-\overline{\mathrm{H}}^{0}}{\mathrm{RT}^{2}}
$$

Observando-se que, em uma mistura, $\overline{\mathrm{H}}^{0}$ é função apenas da temperatura e independe da composição ou pressão, então $\overline{\mathrm{H}}^{0}=\mathrm{H}^{0}$ e a variação de $\ln f$ com a temperatura é dada por:

$$
\left(\frac{\partial \ln \hat{f}^{\mathrm{L}}}{\partial \mathrm{T}}\right)_{\mathrm{P}, \mathrm{x}}=-\frac{\overline{\mathrm{H}}-\mathrm{H}^{0}}{\mathrm{RT}^{2}}
$$

Substituindo-se na Equação 3 as derivadas parciais de fugacidade pelas Equações 4, 7 e 9, e considerando-se que $\mathrm{dx}=0$, ou seja, não há variação de massa no soluto nãovolátil, obtém-se:

$$
\left(\frac{\partial \mathrm{P}}{\partial \mathrm{T}}\right)_{\mathrm{x},[\mathrm{L}-\mathrm{V}]}=\frac{\mathrm{H}^{\mathrm{V}}-\overline{\mathrm{H}^{\mathrm{L}}}}{\mathrm{T}\left(\mathrm{V}^{\mathrm{V}}-\overline{\mathrm{V}^{\mathrm{L}}}\right)}
$$

O numerador da Equação 10 representa a entalpia de vaporização de $1 \mathrm{~mol}$ do solvente em relação a uma solução de composição constante e pode ser expandido adicionando e subtraindo a entalpia do solvente puro $\left(\overline{\mathrm{H}_{2}^{\mathrm{L}}}\right)$, representado pelo subscrito 2, reservando-se o subscrito 1 para o soluto, quando necessário:

$$
\mathrm{H}_{2}^{\mathrm{V}}-\overline{\mathrm{H}_{2}^{\mathrm{L}}}=\left(\mathrm{H}_{2}^{\mathrm{V}}-\mathrm{H}_{2}^{\mathrm{L}}\right)+\left(\mathrm{H}_{2}^{\mathrm{L}}-\overline{\mathrm{H}_{2}^{\mathrm{L}}}\right)
$$

O primeiro termo da Equação 11 representa a entalpia de vaporização do solvente puro $\left(\Delta_{\text {vap }} H\right)$, enquanto o segundo termo refere-se à entalpia molar parcial de mistura $\left(\overline{\Delta \mathrm{H}_{2}^{\mathrm{L}}}\right)$. Como $\left(\Delta_{\text {vap }} \mathrm{H}\right)>\left(\overline{\Delta \mathrm{H}_{2}^{\mathrm{L}}}\right)$, o numerador da Equação 10 pode, geralmente, ser aproximado para a entalpia de vaporização do solvente $\left(\Delta_{\text {vap }} \mathrm{H}\right)^{[40]}$; pode-se, também, assumir que $\left(\mathrm{V}_{2}^{\mathrm{V}}\right)>>\left(\overline{\mathrm{V}_{2}^{\mathrm{L}}}\right)$. Tendo-se em vista que as pressões de operação em um experimento de osmometria de pressão de vapor são bastante reduzidas, pode-se aproximar o comportamento do vapor ao do gás ideal $\left(\mathrm{V}_{2}^{\mathrm{V}}=\mathrm{RT} / \mathrm{P}\right)$. Substituindo-se estas considerações na Equação 10, obtém-se:

$$
\frac{\mathrm{dP}}{\mathrm{dT}}=\frac{\mathrm{P} \Delta_{\mathrm{vap}} \mathrm{H}}{\mathrm{RT}^{2}}
$$

As variações de temperatura e pressão em experimentos de osmometria de pressão de vapor são muito reduzidas, dessa forma, a Equação 12 pode ser reescrita em uma forma aproximada:

$$
\frac{\Delta \mathrm{P}}{\Delta \mathrm{T}}=\frac{\mathrm{P} \Delta_{\mathrm{vap}} \mathrm{H}}{\mathrm{RT}^{2}}
$$

\section{Princípios da Técnica de Osmometria de Pressão de Vapor}

A osmometria de pressão de vapor baseia-se nas propriedades coligativas das soluções e leva em consideração a diferença de pressão de vapor oriunda da presença do soluto em relação ao solvente puro. Trata-se, em princípio, de uma técnica diferencial termoelétrica, uma vez que a propriedade medida é uma pequena variação na temperatura, proveniente de diferentes taxas de evaporação do solvente após o acréscimo de gotas de soluções de concentrações distintas em um termistor que se encontra em equilíbrio sob uma atmosfera saturada pelo vapor do solvente.

O osmômetro é um equipamento dotado de dois circuitos termistores (Figura 1) que sofrem uma variação de resistência proporcional à variação de temperatura, de acordo com a Equação 14:

$$
\Delta \mathrm{R}=\mathrm{R}_{0} \mathrm{a} \Delta \mathrm{T}
$$




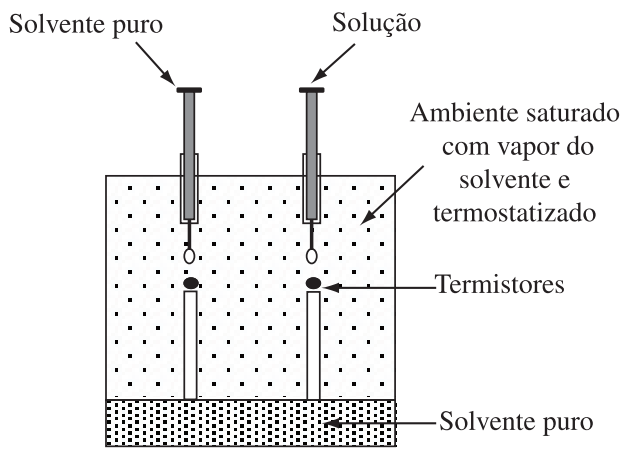

Figura 1. Representação esquemática da câmara de um osmômetro de pressão de vapor.

Em que $\Delta \mathrm{R}$ é a variação da resistência, $\mathrm{R}_{0}$ é uma resistência de referência, a é uma constante de proporcionalidade e $\Delta \mathrm{T}$ é a variação de temperatura.

Em um meio saturado pelo solvente, goteja-se uma pequena quantidade do solvente em um dos termistores e, no outro, da solução do mesmo solvente que satura o meio com conhecida concentração mássica do soluto, cuja massa molar se deseja determinar. Neste experimento, a injeção de uma pequena gota da solução em um dos termistores provoca uma perturbação no equilíbrio termodinâmico e em decorrência do abaixamento da pressão de vapor ocorre a condensação do vapor que satura o meio em torno do volume de solução que circunda o termistor, com consequente queda na temperatura e na resistência. Normalmente, a leitura é obtida por meio da diferença de potencial $(\Delta s)$ em um milivoltímetro, conhecendo-se a corrente elétrica (i) estabelecida:

$$
\Delta \mathrm{s}=\mathrm{i} \Delta \mathrm{R}
$$

e, conseqüentemente, pela Equação 14:

$$
\Delta \mathrm{s}=\mathrm{iR}_{0} \mathrm{a} \Delta \mathrm{T}
$$

Verifica-se, portanto que em um equipamento de osmometria de pressão de vapor uma variação na temperatura é proporcional a uma variação na diferença de potencial elétrico. A Equação 14 pode ser agora incorporada à Equação 13, resultando na equação que relaciona o abaixamento da pressão de vapor do solvente com a diferença de potencial elétrico:

$$
\Delta \mathrm{P}=\mathrm{P}^{0} \frac{\Delta_{\text {vap }} \mathrm{H}}{\mathrm{iR}_{0} \mathrm{aRT}^{2}} \Delta \mathrm{s}
$$

em que o sobrescrito ${ }^{0}$ refere-se ao solvente puro.

Segundo a Lei de Raoult para o equilíbrio líquido-vapor de uma solução ideal:

$$
\mathrm{P}_{2}=\mathrm{P}_{2}^{0}\left(1-\mathrm{x}_{1}\right)
$$

A variação de pressão de vapor do solvente é então obtida pelo rearranjo da Equação 18:

$$
\Delta \mathrm{P}_{2}=\mathrm{P}_{2}^{0} \mathrm{x}_{1}
$$

No caso de soluções muito diluídas, a fração molar em função da quantidade de matéria $(\mathrm{N})$ pode ser aproximada em função das massas $\left(m_{i}\right)$ presentes na solução e das massas molares $\left(\mathrm{M}_{\mathrm{i}}\right)$. Assim:

$$
\mathrm{x}_{1}=\frac{\mathrm{n}_{1}}{\mathrm{n}_{1}+\mathrm{n}_{2}} \cong \frac{\left(\mathrm{m}_{1} / \mathrm{M}_{1}\right)}{\left(\mathrm{m}_{2} / \mathrm{M}_{2}\right)}
$$

em que a aproximação é possível porque $\mathrm{n}_{2}$ é muito maior que $\mathrm{n}_{1}$.

Substituindo-se a Equação 20 na Equação 19, obtém-se:

$$
\Delta \mathrm{P}_{2}=-\mathrm{P}_{2}^{0} \frac{\mathrm{m}_{1} / \mathrm{M}_{1}}{\mathrm{~m}_{2} / \mathrm{M}_{2}}
$$

Substituindo $\Delta \mathrm{P}_{2}$ na Equação 17 e explicitando-se $\Delta \mathrm{s}$, chega-se a:

$$
\Delta \mathrm{s}=-\frac{\mathrm{iR}_{0} \mathrm{aRT}^{2}}{\Delta_{\text {vap }} \mathrm{H}} \frac{\mathrm{m}_{1}}{\mathrm{M}_{1}} \frac{\mathrm{M}_{2}}{\mathrm{~m}_{2}}
$$

Como:

$$
\begin{aligned}
& \mathrm{m}_{2}=\mathrm{V}_{2} \\
& \mathrm{C}=\frac{\mathrm{m}_{1}}{\mathrm{~V}}
\end{aligned}
$$

Substituindo as Equações 23 e 24 na Equação 22, obtémse:

$$
\Delta \mathrm{s}=-\frac{\mathrm{iR}_{0} \mathrm{aRT}^{2}}{\Delta_{\text {vap }} \mathrm{H}} \frac{\mathrm{C}}{\rho_{2}} \frac{\mathrm{M}_{2}}{\mathrm{M}_{1}}
$$

Fazendo:

$$
\mathrm{K}_{\mathrm{i}}=-\mathrm{iR} \mathrm{aRT}^{2} \frac{\mathrm{M}_{2}}{\left(\Delta_{\text {vap }} \mathrm{H} \rho_{2}\right)}
$$

em que $K_{i}$ depende apenas de propriedades do solvente, chega-se à equação:

$$
\Delta \mathrm{s}=\mathrm{K}_{\mathrm{i}} \frac{\mathrm{C}}{\overline{\mathrm{M}_{\mathrm{n}}}}
$$

em que $\bar{M}_{n}$ é a massa molar média do componente que se deseja determinar.

Considerando-se que a variação da tensão é conseqüência de uma variação proporcional da temperatura, a Equação 27 pode ser representada de uma forma mais generalizada como:

$$
(\Delta \mathrm{T})=\mathrm{K}_{\mathrm{a}}\left[\frac{\mathrm{C}}{\overline{\mathrm{M}_{\mathrm{n}}}}+\mathrm{A}_{2} \mathrm{C}^{2}+\mathrm{A}_{3} \mathrm{C}^{3}+\ldots\right]
$$


No estado estacionário, a diferença de temperatura entre os dois termistores é dada pela Equação 28 , na qual $\mathrm{K}_{\mathrm{a}}$ é considerada uma constante de calibração; $\mathrm{C}$ é a concentração e depende das unidades usuais; $\mathrm{A}_{2}$ é o segundo coeficiente do virial, $\mathrm{A}_{3}$ é o terceiro coeficiente do virial, e assim sucessivamente. Para soluções diluídas, ainda que não represente uma garantia de não ocorrer desvios da idealidade, normalmente rearranja-se a Equação 28 e combinando com a Equação 16, obtém-se a Equação 27, que assume a forma na qual é empregada em experimentos de osmometria de pressão de vapor ${ }^{[5]}$.

\section{Cálculo da Massa Molar a Partir de Dados de Osmometria de Pressão de Vapor}

A massa molar de uma substância é obtida indiretamente, por meio de outra substância de massa molar bem determinada usada como referência (padrão primário). As principais características que definem um bom padrão de massa molar são: massa molar conhecida, alto grau de pureza e estabilidade química nas condições experimentais. Além disso, alguns trabalhos ressaltam a importância de se usar, sempre que possível, uma substância de massa molar próxima da amostra que se deseja determinar e com semelhanças químicas ${ }^{[41]}$. Também é recomendável que o padrão seja pouco polidisperso, tendendo a formar soluções monoméricas no solvente utilizado $^{[5]}$.

A constante $\mathrm{K}_{\mathrm{i}}$ que deverá ser obtida para o padrão primário envolve unidades em bases molares e é extraída de curvas $\Delta \mathrm{s}$ (tensão) em função da concentração molal do soluto $\left(\mathrm{W}_{1}\right)$. Assim, para a aplicação da Equação 27, a concentração $\mathrm{C}$ é expressa como $\mathrm{W}_{\mathrm{p}}$ (concentração molal do padrão em mol. $\mathrm{kg}^{-1}$ ) e a massa molar $\overline{\mathrm{M}}_{\mathrm{n}}$ fica inserida na nova constante $\mathrm{K}_{\mathrm{p}}$ (do padrão $\mathrm{p}$ ), de acordo com a equação:

$$
\mathrm{K}_{\mathrm{p}}=\frac{\mathrm{K}_{\mathrm{i}}}{\overline{\mathrm{M}_{\mathrm{n}}}} \frac{1}{\rho}
$$

De forma análoga ao cálculo da constante do padrão primário, é realizado o cálculo da constante $\mathrm{K}_{\mathrm{s}}$ para a substância cuja massa molar se deseja determinar (amostra $\mathrm{S}$ ). Neste caso, porém, a constante envolve unidades mássicas, devido à concentração ser expressa em unidades mássicas ( $\mathrm{X}_{\mathrm{s}} \mathrm{em} \mathrm{g}$ do soluto/kg do solvente).

$$
\mathrm{K}_{\mathrm{S}}=\left(\frac{\Delta \mathrm{s}}{\mathrm{X}_{\mathrm{s}}}\right)_{\mathrm{X}_{\mathrm{S}} \rightarrow 0}
$$

A Equação 30 assume a forma:

$$
\Delta \mathrm{s}=\mathrm{K}_{\mathrm{p}} \mathrm{W}_{\mathrm{p}}
$$

A massa molar pode ser obtida pela aplicação direta da Equação 27 ou pelo cálculo da constante $\mathrm{K}_{\mathrm{p}}$, que pode ser determinada por meio dos dados experimentais através de dois tratamentos:

i) Pela inclinação da curva $\Delta \mathrm{s} \times \mathrm{W}_{\mathrm{p}}$; e ii) Pela extrapolação para diluição infinita $\left(\mathrm{W}_{\mathrm{p}} \rightarrow 0\right)$ da curva $\frac{\Delta \mathrm{s}}{\mathrm{W}_{\mathrm{p}}} \mathrm{xW}_{\mathrm{p}}$; neste caso, a constante é determinada pelo intercepto:

$$
\mathrm{K}_{\mathrm{p}}=\left(\frac{\Delta \mathrm{s}}{\mathrm{W}_{\mathrm{p}}}\right)_{\mathrm{W}_{\mathrm{p}} \rightarrow 0}
$$

As constantes $\mathrm{K}_{\mathrm{p}}$ e $\mathrm{K}_{\mathrm{s}}$ calculadas por meio do intercepto são obtidas de curvas que representam o quociente entre a variação do sinal elétrico e a concentração das espécies em solução em função dessa concentração. Desse modo, considerando-se as mesmas condições de ensaio (mesma temperatura e solvente, baixas concentrações e admitindo que as substâncias em solução produzam espécies moleculares ou iônicas com mesmo grau de dissociação), as constantes devem ser iguais, uma vez que, nestas condições, possuem mesmo número de partículas por unidade de concentração.

Assim, é possível relacionar duas substâncias distintas: a constante obtida para o padrão primário $\left(\mathrm{K}_{\mathrm{p}}\right)$ que já se encontra descrita em termos de número de partículas, por envolver unidades em bases molares, e a constante para a amostra $\left(\mathrm{K}_{\mathrm{s}}\right)$, que envolve unidades mássicas.

A massa molar é obtida pela razão entre estas duas constantes, a constante do padrão em kg.mol ${ }^{-1}$ e a constante da amostra em kg.g ${ }^{-1}$, conforme a equação:

$$
\overline{\mathrm{M}_{\mathrm{n}}}=\frac{\mathrm{K}_{\mathrm{p}}}{\mathrm{K}_{\mathrm{s}}}\left(\frac{\mathrm{g}}{\mathrm{mol}}\right)
$$

O método de determinação da constante (ii) é o mais tradicionalmente usado em experimentos envolvendo propriedades coligativas, entretanto, Collins et al. ${ }^{[42]}$ mostraram que as curvas exibidas na forma $\left(\frac{\text { propriedade }}{\text { concentração }} x\right.$ concentração $)$, levam a um espalhamento significativo dos pontos experimentais, o que afeta o cálculo da constante. Sabadini et al. ${ }^{[43]}$, propuseram o método (i) como um procedimento alternativo e compararam os dois procedimentos através da determinação de massas molares de uma série de polímeros do grupo dos polietilenos glicois (PEG) de diversos tamanhos.

As curvas dos polietilenos glicois obtidas em experimentos de OPV em baixas concentrações apresentaram um comportamento linear, sugerindo que a constante pode ser extraída diretamente da inclinação destas curvas em gráficos $\Delta \mathrm{s} x$ concentração do polímero, e a massa molar é obtida da razão entre estas duas novas constantes. Ambas as alternativas foram comparadas e observou-se que para os seis polímeros estudados foram encontrados valores de $\overline{\mathrm{M}}_{\mathrm{n}}$ mais próximos dos valores fornecidos pelo fabricante quando se emprega $o$ método alternativo.

Neste trabalho, busca-se analisar, comparativamente, os principais métodos de determinação de massa molar através das curvas construídas a partir de dados de osmometria de 
pressão de vapor e propor um tratamento que permita obter valores mais precisos para a massa molar de asfaltenos.

\section{Experimental}

Ramos $^{[5]}$ extraiu frações asfaltênicas C5I (insolúveis em n-pentano) e C7I (insolúveis em n-heptano) de amostras de petróleos oriundas do Campo de Marlim - Bacia de Campos/ RJ através do procedimento IP143/84 ${ }^{[7]}$ e preparou soluções em tolueno (Merck) com diversas concentrações de cada fração. Soluções de dibenzoíla (Kodak) e polietileno glicol 3350 (PEG 3350) (Aldrich) foram também preparadas em tolueno nas mesmas concentrações.

Foram então executados experimentos de osmometria de pressão de vapor, que forneceram as variações de resistência decorrentes da injeção de cada solução. Estes dados permitiram a obtenção das variações de tensão correspondentes e a construção de gráficos envolvendo tensão e concentração de cada composto em análise, com base no desenvolvimento prático-teórico descrito nas Seções 3 e 4. Em seguida, partindo destas curvas e utilizando a metodologia proposta por Sabadini et al. ${ }^{[43]}$, Ramos $^{[5]}$ calculou a massa molar dos asfaltenos C5I e C7I.

Neste estudo, os dados experimentais assim obtidos por $\operatorname{Ramos}^{[5]}$ são apresentados e trabalhados de modo alternativo.

\section{Resultados e Discussão}

A primeira etapa para a determinação da massa molar dos asfaltenos consistiu na escolha do padrão primário. O tolueno é um bom solvente para asfaltenos e a substância usada como padrão primário em tolueno, segundo especificações do equipamento, foi a dibenzoíla, que, no entanto, possui massa molar muito inferior à estimada para asfaltenos. Este fato sugeriu o uso de uma substância de massa molar entre 1.000 e 10.000 g.mol ${ }^{-1}$, de acordo com os valores de massa molar para asfaltenos relatados na literatura, para a aferição da dibenzoíla. Os polietilenos glicois apresentam-se como sistemas pouco polidispersos em tolueno ${ }^{[43]}$, além de valores de massa molar mais próxima a dos asfaltenos, quando comparado à dibenzoíla, e, desta forma, o PEG 3350 foi selecionado como substância de referência.

Ramos $^{[5]}$ adotou, assim, um procedimento alternativo, visando obter valores mais confiáveis. Como o padrão primário foi aprovado na determinação da massa molar do PEG 3350, foi também utilizado para a determinação da massa molar dos asfaltenos.

Inicialmente, construiu-se a curva de calibração utilizando-se a dibenzoíla como padrão primário. A curva obtida é exibida na Figura 2 e mostra a tensão lida no osmômetro de pressão de vapor como função da concentração de dibenzoíla em tolueno. Todos os experimentos foram realizados com três repetições independentes e cada ponto experimental representa a média de pelo menos cinco determinações.
A constante de calibração foi calculada, em princípio, utilizando o método proposto por Sabadini et al. ${ }^{[43]}$, ou seja, através dos coeficientes angulares das curvas $\Delta \mathrm{s} x$ concentração. As constantes $\mathrm{K}_{\mathrm{s}}$ serão relatadas com a subscrito 1 para o método alternativo, proposto por Sabadini et al. ${ }^{[43]} ; 2$ para o convencional; e 3 para o proposto neste trabalho.

Da curva exibida na Figura 2 o cálculo da constante resultou em $\mathrm{K}_{1}=1299 \pm 7 \mathrm{mV} \mathrm{kg} \cdot \mathrm{mol}^{-1}$.

Na Figura 3 é apresentada a curva do quociente entre tensão e concentração como função da concentração de dibenzoíla. Pelo procedimento convencional, obteve-se a constante $\mathrm{K}_{2}=1542 \mathrm{~kg} \cdot \mathrm{mol}^{-1} \mathrm{mV}$ e $\mathrm{R}=0,93169$, verificando, conforme observado por Sabadini et al. ${ }^{[43]}$, maior dispersão dos pontos para um ajuste linear.

A Figura 4 exibe a curva da tensão como função da concentração mássica do PEG 3350, através da qual se obteve um

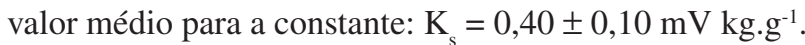

A razão entre as constantes $\overline{\mathrm{M}}_{\mathrm{n}}=\left(\mathrm{K}_{\mathrm{p}} / \mathrm{K}_{\mathrm{s}}\right)$, forneceu a massa molar 3246 g. mol $^{-1}$ para o PEG 3350, o que representa um valor bastante consistente, com um desvio de $3 \%$ em relação à massa molar de $3350 \mathrm{~g} \cdot \mathrm{mol}^{-1}$ relatada pelo fabricante.

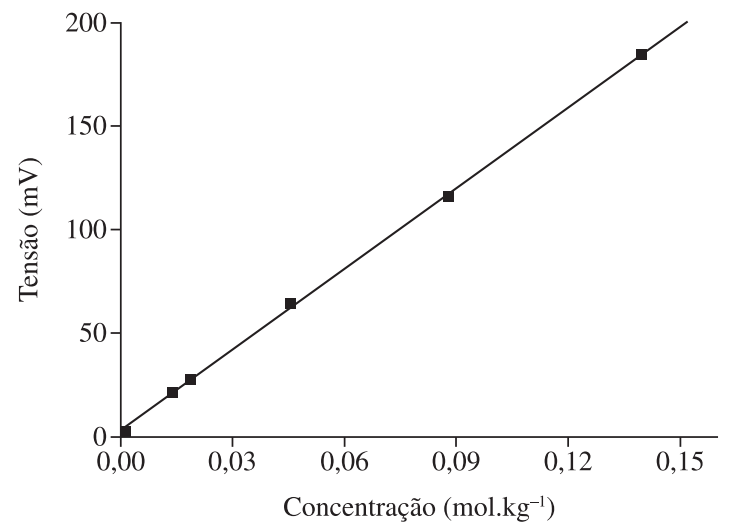

Figura 2. Tensão como função da concentração de dibenzoíla em tolueno a $65{ }^{\circ} \mathrm{C}$. Curva de calibração - Padrão primário (dibenzoíla $\left.\mathrm{MM}=210,23 \mathrm{~g} \cdot \mathrm{mol}^{-1}\right)$. Ajuste linear com equação $\mathrm{T}=1295,1995 \mathrm{C}+3,0783$ e $\mathrm{R}=0,99956$.

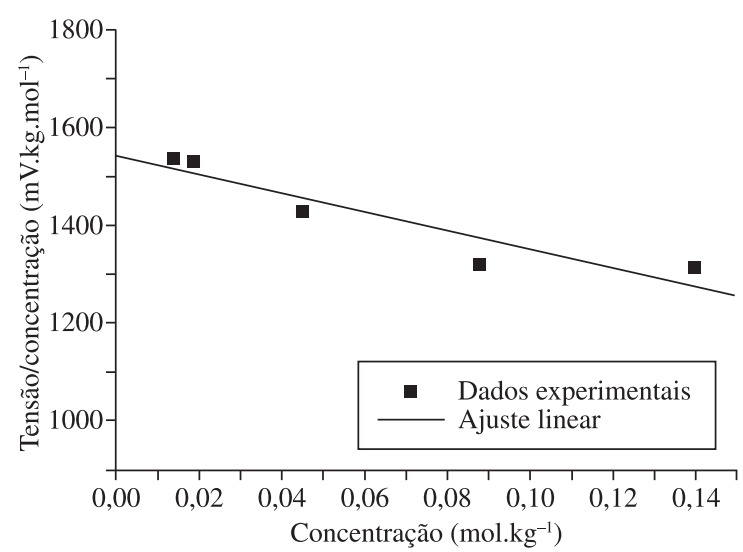

Figura 3. Quociente entre tensão e concentração como função da concentração de benzoíla. Ajuste linear com equação $(T / C)=-1908,7842$ $\mathrm{C}+1542,1074$ e $\mathrm{R}=0,93169$. 
Sabadini et al ${ }^{[43]}$ apresentaram erros entre 4 e $5 \%$ nessa faixa de massa molecular para o PEG.

No caso de uma aproximação de até $5 \%$ entre o valor da massa molar obtida para o PEG 3350 neste experimento utilizando a dibenzoíla como referência e o valor relatado pelo fabricante, os dados obtidos para a dibenzoíla seriam utilizados para a determinação da massa molar dos asfaltenos. $\mathrm{O}$ valor de 5\% foi tomado arbitrariamente como um parâmetro que estabelece apenas um critério comparativo em relação ao valor relatado pelo fabricante. O resultado positivo da constante de calibração da dibenzoíla obtida pelo método alternativo $\left(\mathrm{K}_{1}\right)$ permitiu sua aplicação nos cálculos subseqüentes para determinação da massa molar dos asfaltenos.

A Figura 5 apresenta a curva construída a partir do quociente entre a tensão e a concentração como função da concentração de PEG 3350, utilizada na obtenção de $\mathrm{K}_{2}$.

Do tratamento convencional da curva de calibração do PEG 3350 (Figura 5), obteve-se $\mathrm{K}_{2}=0,4062 \mathrm{mV} \mathrm{kg. \textrm {mol } ^ { - 1 }}$ e $\mathrm{R}=0,18157$, o que indica uma grande dispersão entre os pontos; não obstante, a massa molar para o PEG 3350 foi calculada, resultando em 3796 g.mol ${ }^{-1}$, o que representa um desvio de $13 \%$ em relação à massa molar relatada.

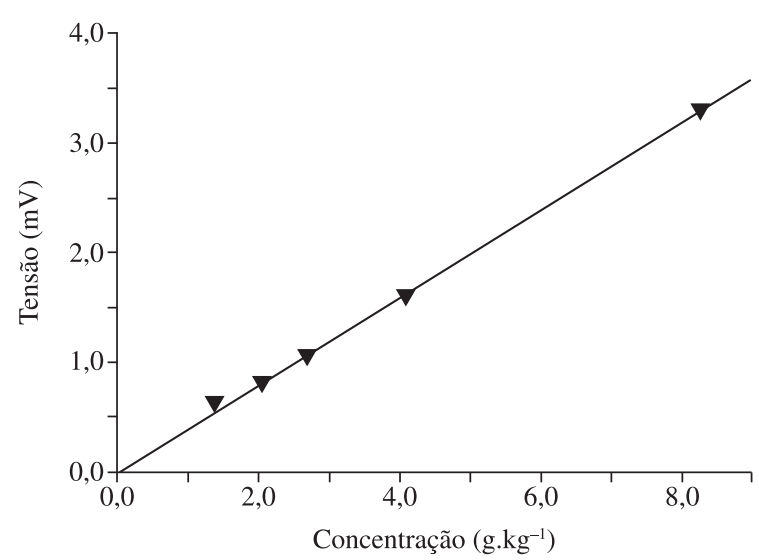

Figura 4. Tensão como função da concentração de PEG 3350 em tolueno a $65{ }^{\circ} \mathrm{C}$. Ajuste linear com equação $\mathrm{T}=0,3995 \mathrm{C}-0,00641$ e $\mathrm{R}=0,99928$.

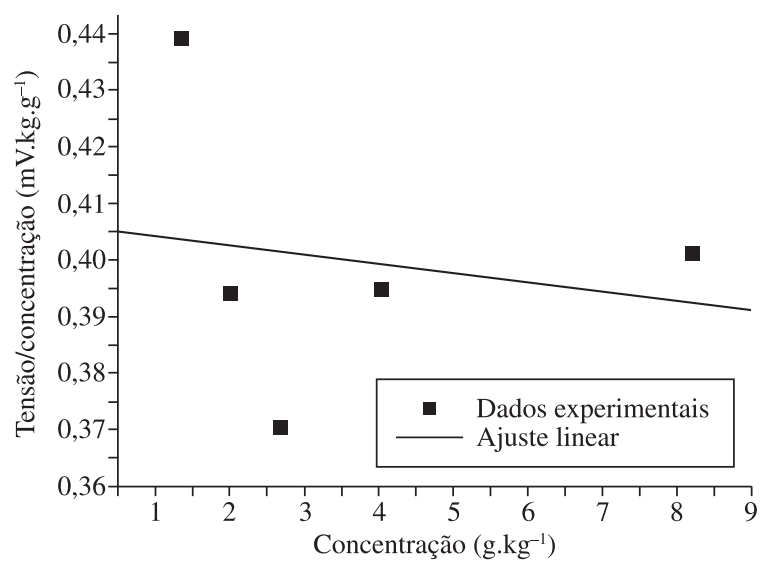

Figura 5. Quociente entre tensão e concentração como função da concentração de PEG 3350 em tolueno a $65{ }^{\circ} \mathrm{C}$. Ajuste linear com equação $(\mathrm{T} / \mathrm{C})=-0,0017 \mathrm{C}+0,4062$ e $\mathrm{R}=0,18157$.
A Figura 6 contém as medidas de tensão em função da concentração mássica dos asfaltenos C5I. A concentração dos asfaltenos em tolueno foi avaliada em uma faixa ampla de forma a englobar a concentração micelar crítica destes asfaltenos $\left(15,4\right.$ g. $\mathrm{L}^{-1}$ em tolueno e obtida de medidas de tensão superficial a $25{ }^{\circ} \mathrm{C}$, equivalente a aproximadamente $17,7 \mathrm{~g} . \mathrm{kg}^{-1}$, considerando a densidade do tolueno de $0,87 \mathrm{~kg} . \mathrm{L}^{-1}$, fornecida pelo fabricante).

A Figura 7 exibe a curva obtida através do quociente entre a tensão e a concentração como função da concentração de asfaltenos C5I. Em soluções muito diluídas o sinal da tensão é muito baixo, sobretudo considerando-se macromoléculas que, neste caso, geram poucas partículas em solução, provocando um desvio da tendência geral observada nas soluções mais concentradas. Apesar disso, a constante $\mathrm{K}_{2}=0,2963$ foi obtida a partir desta curva.

Em seguida, os primeiros dois pontos da curva apresentada na Figura 7 foram rejeitados e construiu-se um novo gráfico do quociente entre tensão e concentração como função da concentração de asfaltenos C5I, no qual se observa um melhor ajuste linear, como mostra a Figura 8, e que produziu a constante $\mathrm{K}_{2}{ }^{\prime}=0,40874$ para determinação da massa molar pelo método convencional. Embora um coeficiente

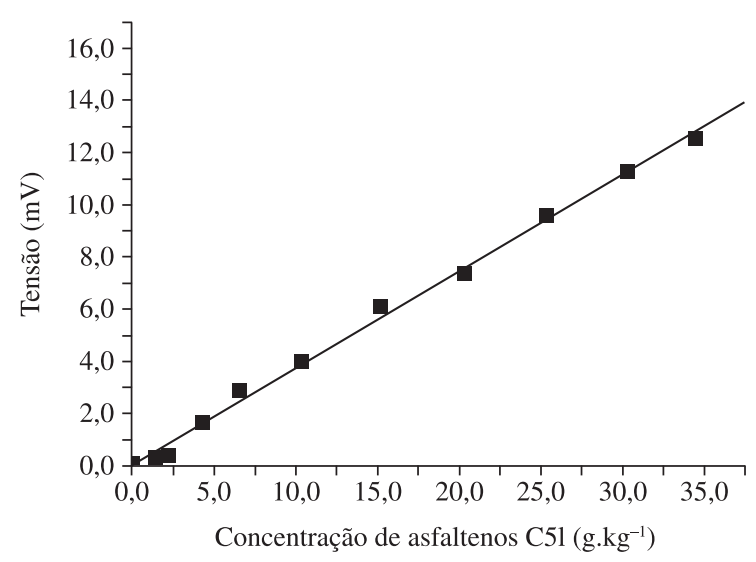

Figura 6. Tensão em função da concentração de asfaltenos C5I em tolueno a $65{ }^{\circ} \mathrm{C}$. Ajuste linear com equação $(\mathrm{T} / \mathrm{C})=-0,0017 \mathrm{C}+0,4062 \mathrm{e}$ $\mathrm{R}=0,18157$.

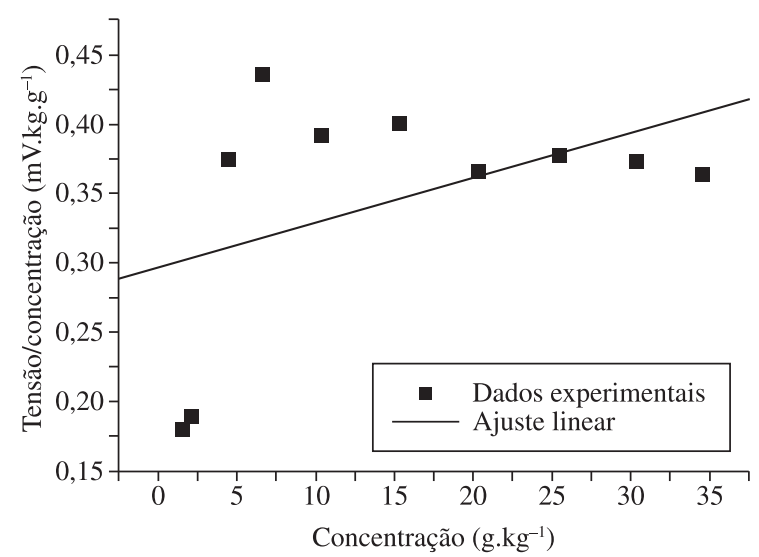

Figura 7. Quociente entre tensão e concentração como função da concentração de asfaltenos C5I. Ajuste linear com equação $(T / C)=0,0032 C+0,2963$ e $\mathrm{R}=0,44614$. 
de correlação igual a 0,36361 indique pouca adequação da curva ao modelo polinomial de segunda ordem, a constante $\mathrm{K}_{3}=0,4058$ foi obtida e também usada no cálculo da massa molar destes asfaltenos.

A curva construída para os asfaltenos C7I com procedimento semelhante ao aplicado aos C5I é apresentada na Figura 9. Analogamente, a concentração foi avaliada de modo a abranger a concentração micelar crítica destes asfaltenos em tolueno $\left(6,5\right.$ g.L. $\mathrm{L}^{-1}$ ou $7,47 \mathrm{~g} . \mathrm{kg}^{-1}$, a $\left.25^{\circ} \mathrm{C}\right)$. Verificase na Figura 9 um desvio de comportamento na concentração de 10,0 g. $\mathrm{kg}^{-1}$ consistente com um processo agregativo em solução e, portanto, esse resultado reforça os dados de tensão superficial que indicam a formação de micelas próximo dessa concentração.

Dessa forma, preferiu-se trabalhar em concentrações menores que $10 \mathrm{~g} . \mathrm{kg}^{-1}$, assumindo, em princípio, ser este o limite para ocorrência do primeiro fenômeno crítico na formação dos agregados asfaltênicos no meio.

Nas Figuras 10 e 11 verificam-se gráficos do quociente entre tensão e concentração como função da concentração de asfaltenos C7I. Na Figura 10 é mostrada a regressão linear,

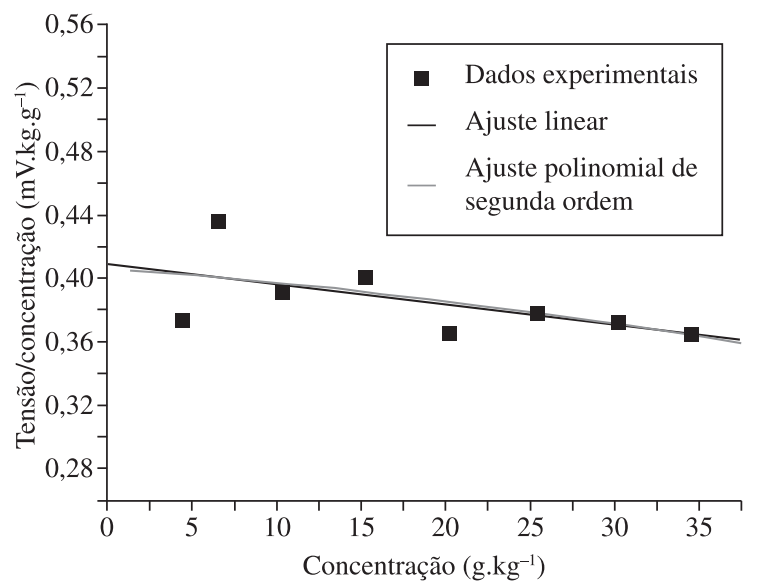

Figura 8. Quociente entre tensão e concentração como função da concentração de asfaltenos C5I. Ajuste linear com equação $(T / C)=-0,0013 \mathrm{C}+$ 0,4087 e $\mathrm{R}=0,60143$. Ajuste polinomial de segunda ordem com equação $(\mathrm{T} / \mathrm{C})=0,4058-8,4920 \cdot 10^{-4} \mathrm{C}-1,1537 \cdot 10^{-5} \mathrm{C}^{2}$ e $\mathrm{R}^{\prime}=0,36361$

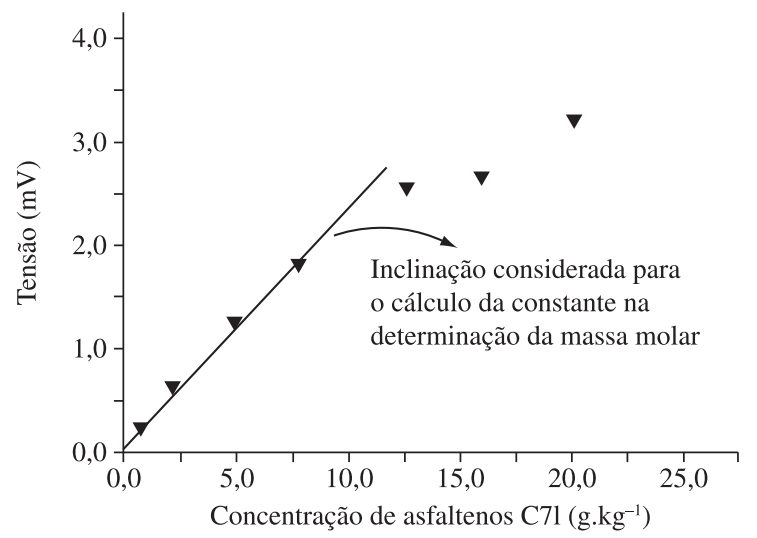

Figura 9. Tensão em função da concentração de asfaltenos C7I em tolueno a $65^{\circ} \mathrm{C}$.

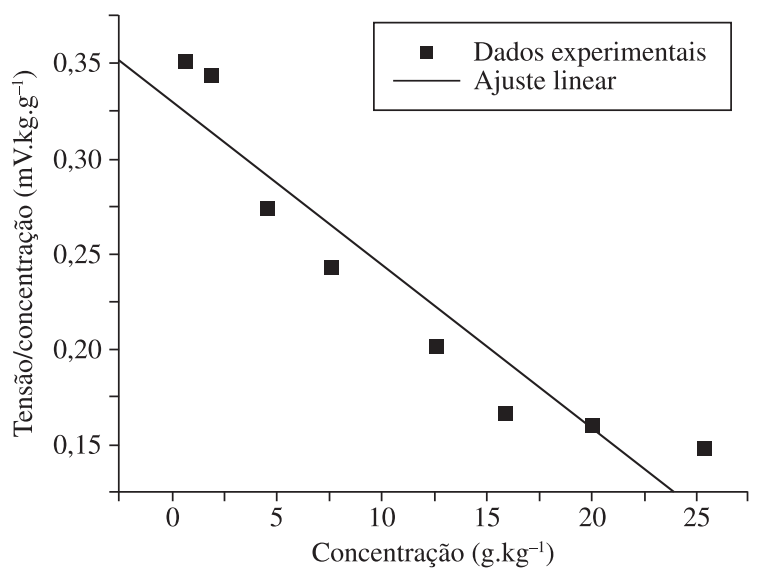

Figura 10. Quociente entre tensão e concentração como função da concentração de asfaltenos C7I. Ajuste linear com equação $(\mathrm{T} / \mathrm{C})=-0,0086 \mathrm{C}+0,3304$ e $\mathrm{R}=0,94645$.

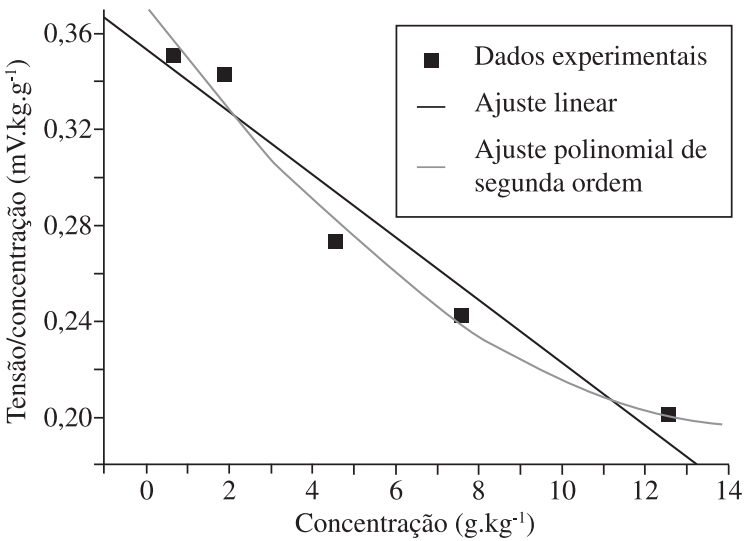

Figura 11. Quociente entre tensão e concentração como função da concentração de asfaltenos C7I. Regressão linear com equação $(\mathrm{T} / \mathrm{C})=-0,0131 \mathrm{C}$ $+0,3534$ e $\mathrm{R}=0,97173$. Ajuste polinomial de segunda ordem com equação $(\mathrm{T} / \mathrm{C})=0,3721-0,0233 \mathrm{C}+7,7163 \cdot 10^{-4} \mathrm{C}^{2}$ e $\mathrm{R}^{\prime}=0,98383$.

com todos os pontos obtidos a partir da Figura 9, que forneceu a constante $\mathrm{K}_{2}=0,3304$.

A Figura 11 apresenta somente os cinco primeiros pontos obtidos da primeira inclinação da curva da Figura 9, levados em consideração para o cálculo de $\mathrm{K}_{1}$; neste caso, obteve-se a constante $\mathrm{K}_{2}{ }^{\prime}=0,3534$ para o cálculo da massa molar pelo método tradicional e ajuste linear, mas observou-se uma melhor adequação da curva ao modelo polinomial e a constante $\mathrm{K}_{3}=0,3721$ foi determinada.

Observa-se na Figura 10 que, assim como aconteceu com os asfaltenos C5I, os dois primeiros pontos representam soluções muito diluídas, cujo sinal da tensão é baixo, e há um desvio da tendência geral observada nas soluções mais concentradas. Além disso, pode-se considerar que os dois últimos pontos também representam um desvio, uma vez que os mesmos foram obtidos a partir de concentrações muito superiores à concentração micelar crítica dos asfaltenos C7I e há maior possibilidade de estar ocorrendo auto-associação, pois estes asfaltenos apresentam massa molar média mais elevada que a dos $\mathrm{C} 5 \mathrm{I}^{[5]}$. 
Assim, os quatro pontos considerados inadequados foram excluídos e obteve-se o gráfico apresentada na Figura 12. O ajuste linear forneceu a constante $\mathrm{K}_{2} "=0,3534$, enquanto o ajuste polinomial forneceu $\mathrm{K}_{3}{ }^{\prime}=0,3138$.

A curva da dibenzoíla (Figura 2) apresentou um bom ajuste linear, mas a curva do PEG 3350 (Figura 4) apresentou um espalhamento maior dos pontos experimentais quando ajustados para um comportamento linear. A diferença entre estes sistemas deve-se principalmente ao fato de o PEG 3350 exibir maior grau de polidispersão, comportamento característico de sistemas poliméricos.

Para as curvas referentes aos sistemas contendo asfaltenos verificou-se um espalhamento bem maior dos pontos quando ajustados para um comportamento linear. A curva dos asfaltenos C7I exibe uma curvatura mais acentuada que a dos asfaltenos C5I, o que permitiu um melhor ajuste a um modelo de comportamento polinomial. Este comportamento pode refletir a diminuição do número de espécies no seio da solução, por exemplo, pela formação de agregados. Para os asfaltenos C7I (Figura 9), a constante foi calculada considerando os pontos experimentais até uma concentração de aproximadamente $10 \mathrm{~g} \cdot \mathrm{kg}^{-1}$, faixa que define melhor um comportamento linear. Este procedimento encontra-se em concordância com a metodologia proposta por Sabadini et al..$^{[43]}$, que consideram menores os erros dos valores de massa molar obtidos por osmometria de pressão de vapor quando calculados a con-

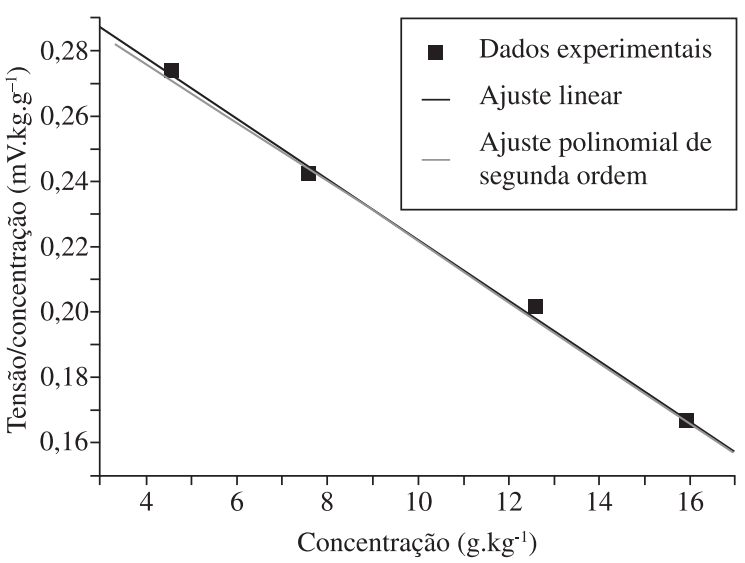

Figura 12. Quociente entre tensão e concentração como função da concentração de asfaltenos C7I. Regressão linear com equação $(T / C)=-0,0092 \mathrm{C}$ $+0,3145$ e $R=0,99896$. Ajuste polinomial de segunda ordem com equação $(T / C)=0,3138-0,0090 \mathrm{C}-1,3633 \cdot 10^{-5} \mathrm{C}^{2}$ e R' $=0,99793$. centrações relativamente baixas e na faixa em que os pontos experimentais se apresentam como uma reta.

As massas molares médias numéricas dos asfaltenos C5I e C7I foram determinadas das curvas apresentadas nas Figuras 6, 8, 11 e 12 e relatadas na Tabela 1, utilizando os mesmos procedimentos descritos anteriormente para determinação da massa molar do PEG 3350.

Os asfaltenos C5I apresentaram um menor valor de massa molar comparado aos asfaltenos C7I, através de todos os tratamentos, exceto quando empregado o método tradicional e levando em consideração todos os valores obtidos experimentalmente.

Assumindo que os asfaltenos são sistemas polidispersos, este resultado indica que estruturalmente os asfaltenos C5I são constituídos de uma fração ponderal maior de moléculas de menor massa molar, provavelmente resinas.

O resultado obtido com a metodologia proposta por Sabadini et al..$^{[43]}$, encontra-se consistente com os obtidos pelo método SARA, que indica que os asfaltenos C5I apresentam em seu conteúdo uma quantidade maior de resinas. Os valores de massa molar determinados para os asfaltenos C5I e C7I situaram-se dentro da faixa de massas molares relatados em diversos trabalhos ${ }^{[5]} \mathrm{e}$ não diferiram muito destes.

A massa molar dos asfaltenos C5I, obtida pela metodologia discutida, isto é, uma combinação do método convencional com tratamentos adicionais dos dados, que, neste caso, foram exclusão dos dois primeiros pontos e ajuste polinomial, foi de 3800 g.mol ${ }^{-1}$, com um erro de $17 \%$ em relação àquela obtida pelo método proposto por Sabadini et al. ${ }^{[43]}$. O processo de obtenção da massa molar dos asfaltenos C7I distingue-se daquele aplicado aos asfaltenos C5I pela exclusão, neste caso, também dos dois últimos pontos e a massa molar obtida foi de 4914 g.mol ${ }^{-1}$, com um erro relativo de $10 \%$.

\section{Conclusão}

Os diversos tratamentos aplicados aos dados experimentais de osmometria de pressão de vapor obtidos de soluções de asfaltenos em tolueno em concentrações abaixo e um pouco acima das concentrações micelares críticas forneceram valores entre $3200-5200$ g. $\mathrm{mol}^{-1}$ para os asfaltenos C5I e entre 4100-5400 g.mol ${ }^{-1}$ para os asfaltenos C7I. Estes valores concordam com os relatados na literatura para estas macromoléculas do petróleo. Em geral, os resultados de massa

Tabela 1. Massa molar média numérica dos Asfaltenos C5I e C7I calculadas a partir de dados de Osmometria de Pressão de Vapor, a $65{ }^{\circ} \mathrm{C}$ e usando tolueno como solvente nas determinações.

\begin{tabular}{|c|c|c|}
\hline Asfaltenos & C5I & C7I \\
\hline Método & \multicolumn{2}{|c|}{ Massa molar (g.mol $\left.{ }^{-1}\right)$} \\
\hline Proposto por Sabadini et al. ${ }^{[22]}$ & $3250 \pm 150$ & $5430 \pm 270$ \\
\hline Convencional (todos os pontos e ajuste linear) & 5205 & 4666 \\
\hline Convencional (pontos selecionados e ajuste linear) & 3773 & 4363 \\
\hline Convencional (pontos selecionados e ajuste polinomial) & 3800 & 4144 \\
\hline Convencional (pontos intermediários e ajuste linear) & - & 4896 \\
\hline Convencional (pontos intermediários e ajuste polinomial) & - & 4914 \\
\hline
\end{tabular}


molar dos asfaltenos C5I foram inferiores aos dos asfaltenos C7I confirmando que a fração C5I possui em sua composição moléculas com menores valores de massa molar.

Com relação aos diversos tratamentos empregados no cálculo da massa molar, o procedimento avaliado neste trabalho, excluindo pontos experimentais na região de baixa sensibilidade do equipamento e levando em consideração, tanto quanto possível, a tendência das curvas em diluições infinitas, permitiu a obtenção de valores de massa molar, em princípio, mais confiáveis.

Verifica-se ainda, porém, a necessidade da realização de estudos posteriores empregando sistemas de massa molar bem determinada para uma comprovação mais sustentável deste procedimento.

\section{Agradecimentos}

Ao Professor Watson Loh, do Instituto de Química/ UNICAMP, pelas contribuições na construção deste trabalho.

\section{Referências Bibliográficas}

1. Yarranton, H. W. \& Masliyah, J. H. - AIChE Journal, 42, p.3533-3543 (1996).

2. Misra, S.; Baruah, S. \& Singh, K. - SPE Production \& Facilities, 10, p.50-54 (1995).

3. King, S. R. - JTP, 48, p.723-724 (1996).

4. Mohamed, R. S.; Loh, W. \& Ramos, A. C. S. - Energy Fuels, 13, p.323-327 (1999).

5. Ramos, A. C. S. - "Asfaltenos em petróleos brasileiros: agregação em solventes aromáticos, desenvolvimento de aditivos e estabilização de emulsões", Tese de Doutorado, Universidade Estadual de Campinas, Brasil (2001).

6. Garreto, M. S. E. - “Adsorção de asfaltenos sobre os sólidos carvão ativado, quitosana e sílica", Monografia de Graduação, Universidade Federal do Maranhão, Brasil (2003).

7. Institute of Petroleum. IP143/84. "Standard methods for analysis and testing of petroleum and related products". John Wiley \& Sons (1989).

8. Costa, A. C. - "Formação e estabilização de emulsões de petróleo em sistema modelo: efeito de moléculas de resinas $e$ asfaltenos e de propriedades específicas entre as fases aquosa e orgânica", Dissertação de Mestrado, Universidade Federal do Maranhão, Brasil (2004).

9. Speight, J. G. - Revue de l'Institute Français du Pétrole, 40, p.51-61 (1985).

10. Mustafa, M. F.; Al-Jarrah, M. M. F. \& Apikian, R. L. - J. Chem. Technol. Biotechnol., 39, p.231-236 (1987).

11. Yen, T. F. - "Multiple structural orders of asphaltenes", in: Aphaltenes and Asphalts, T. F. Yen, \& G. V. Chiligarian (Eds.), Elsevier Science, New York (1994).

12. Fuhr, B. J.; Cathea, C.; Coates, L.; Kalra, H. \& Majeed, A. I. - Fuel, 70, p.1293-1297 (1991).

13. Acevedo, S.; Escobar, G.; Gutiérrez, L. B. \& D’Aquino, J. Fuel, 71, p.1077-1079 (1992).

14. Larson, J. W. \& Choudry, P. J. - J. Org. Chem., 44, p.28562859 (1979).

15. Al-Jarrah, M. M. F.; Al-Soufi, H. H.; Apikian, R. L.; Al-Saleem, H. \& Naoom, S. S. - Fuel Sci. Technol., 4, p.249-260 (1986).

16. Wiehe, I. A. - J. Dispersion Sci. Technol., 28, p.431-435 (2007).
17. Pereira, J. C.; Luis, M. A. \& Cubillos, S. P. - Pet. Sci. Technol., 26, p.181-190 (2008).

18. Acevedo, S.; Gutierrez, L. B.; Negrin, G. \& Pereira, J. C. Energy Fuels, 19, p.1548-1560 (2005).

19. Moschopedis, S. E.; Fryer, J. F. \& Speight, J. G. - Fuel, 55, p.227-232 (1976).

20. Al-Jarrah, M. M. F. \& Apikian, R. L. - J. Chem. Technol. Biotechnol, 39, p.231-236 (1987).

21. Al-Jarrah, M. M. F. \& Al-Dujaili, A. H. - Fuel Sci. Technol., 7, p.69-88 (1989).

22. Badre, S.; Gonçalves, C.; Norinaga, K.; Gustavson, G. \& Mullins, O. C. - Fuel, 85, p.1-11 (2006).

23. Mullins, O. C. - Fuel, 86, p.309-312 (2007).

24. Mullins, O. C.; Martínez-haya, B. \& Marshall, A. G. - Energy Fuels, 22, p.1765-1773 (2008).

25. Storm, D. A.; Sheu, E. Y. \& Detar, M. M. - Fuel, 72, p.977-981 (1992).

26. Sirota, E. B. - Energy Fuels, 19, p.1290-1296 (2005).

27. Monte, M. B. M.; Coelho, R. R. \& Middea, A. - "Investigation of molecular weight and aggregation of asphaltenes in organic solvents using surface tension measurements", in: 2002 International Conference on Heavy Organic Depositions - Proceedings - HOD 2002, Jalisco - Mexico, dez (2002).

28. Rogel, E.; León, O.; Torres, G. \& Espidel, J. - Fuel, 79, p.1389-1394 (2000).

29. Merino-Garcia, D. \& Andersen, S. I. - J. Dispersion Sci. Technol., 26, p.217-225 (2005).

30. Guzman, A.; Bueno, A. \& Carbognani, L. - Pet. Sci. Technol., 27, p.801-816 (2009).

31. Gonçalves, S.; Castillo, J.; Fernández, A. \& Hung, J. - Fuel, 83, p.1823-1828 (2005).

32. Andreatta, G.; Bostrom, N. \& Mullins, O. C. - Langmuir, 21, p.2728-2736 (2005).

33. Mostwfi, F.; Indo, K.; Mullins, O. C. \& Mcfarlane, R. - Energy Fuels, 23, p.1194-1200 (2009).

34. Wargadalam, V. J.; Norinaga, K. \& Iino, M. - Fuel, 81, p.1403-1407 (2002).

35. Schneider, M. H.; Andrews, A. B.; Mitra-Kirtley, S. \& Mullins, O. C. - Energy Fuels, 21, p.2875-2882 (2007).

36. Guerra, R. E.; Ladavac, K.; Andrews, A. B.; Mullins, O. C. \& Sem, P. N. - Fuel, 86, p.2016-2020 (2007).

37. Lisitza, N. V.; Freed, D. E.; Sen, P. N. \& Song, Y. Q. - Energy Fuels, 23, p.1189-1193 (2009).

38. Betancourt, S. S.; Ventura, G. T.; Pomerantz, A. E.; Viloria, O.; Dubost, F. X.; Zuo, J.; Monson, G.; Bustamante, D.; Purcell, J. M.; Nelson, R. K.; Rodgers, R. P.; Reddy, C. M.; Marshall, A. G. \& Mullins, O. C. - Energy Fuels, 23, p.1178-1188 (2009).

39. Vazquez, D \& Mansoori, G. A. - J. Pet. Sci. Eng., 26, p.49-55 (2000).

40. Modell, M. \& Reid, R. C. - "Thermodynamics and its applications", Prentice-Hall PTR, Englewood Cliffs (1983).

41. Lucas, E. F.; Soares, B. G. \& Monteiro, E. E. C. - "Caracterização de polímeros, Determinação de peso molecular e análise térmica", E-papers, Rio de Janeiro (2001).

42. Collins, E. A.; Bares, J. \& Bilmayer, F. W. - "Experiments in polymer sciences", John-Wiley, New York (1973).

43. Sabadini, E.; Assano, E. M. \& Atvars, T. D. Z. - Inc. J. Appl Polym Sci, 65, p.595-600 (1997).

Enviado: $26 / 08 / 08$

Reenviado: 05/06/09

Aceito: $17 / 06 / 09$ 